\title{
Training bei Brustkrebs: Je intensiver, desto effektiver
}

\author{
Schon mehrfach wurden günstige Wirkungen körperlichen Trainings bei \\ Brustkrebspatientinnen unter adjuvanter Therapie beschrieben. Jetzt gab es \\ einen direkten Vergleich eines intensiveren Trainings mit einem leichteren.
}

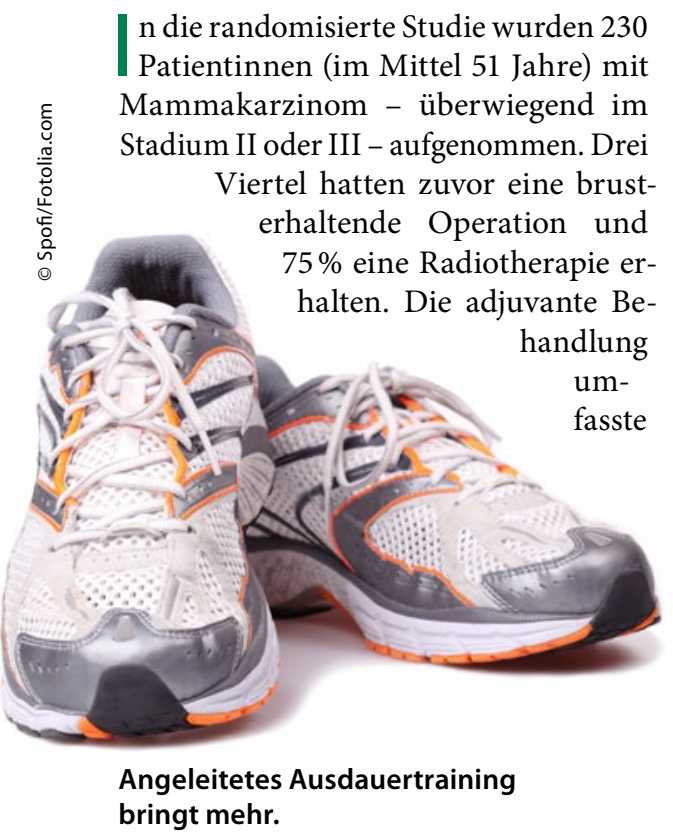

u. a. Anthrazykline und Taxane. Rund ein Drittel der Patientinnen erhielt ein selbstorganisiertes, leichtes häusliches Training (Onco-Move). Ein Drittel vollzog ein mäßig bis sehr intensives kombiniertes Ausdauer- und aerobes Training unter Anleitung eines speziell ausgebildeten Physiotherapeuten (OnTrack). Ein Drittel bekam die normale Betreuung im jeweiligen Zentrum. Vor Beginn der adjuvanten Therapie, am Ende und 6 Monate danach wurden mithilfe verschiedener Tests die körperliche Leistungsfähigkeit ermittelt und per Fragebogen die Ergebnisse aus Sicht der Patientinnen erhoben.

Im Vergleich zur Kontrollgruppe wurden unter Onco-Move und OnTrack eine geringere Abnahme der kardiorespiratorischen Fitness $(p<0,001)$, bessere körperliche Funktionen ( $p \leq 0,001)$, we- niger Übelkeit und Erbrechen ( $\mathrm{p}=0,029$ bzw. 0,031) und weniger Schmerzen ( $\mathrm{p}=0,003$ bzw. 0,011) dokumentiert. OnTrack führte darüber hinaus zu besseren Ergebnissen bei der Muskelstärke $(\mathrm{p}=0,002)$ und weniger physischer Fatigue $(\mathrm{p}<0,001)$. Darunter war auch bei weniger Patientinnen eine Dosisanpassung der Chemotherapie erforderlich.

Fazit: Ein mäßig bis sehr intensives kombiniertes Programm aus Ausdauer- und aerobem Training unter Anleitung ist für Patientinnen mit einer adjuvanten Therapie am wirkungsvollsten. Ein weniger anspruchsvolles körperliches Training ist jedoch eine gangbare Alternative für Frauen, die nicht in der Lage oder willens sind, ein intensiveres Programm durchzuführen.

Brigitte Schalhorn

van Waart $\mathrm{H}$ et al. Effect of low-intensity physical activity and moderate- to high-intensity physical exercise during adjuvant chemotherapy on physical fitness, fatigue, and chemotherapy completion rates: results of the PACES randomized clinical trial. J Clin Oncol. 2015;33(17):1918-27.

\section{Chemotoxizitätsrezidive bei Senioren: Risiko- faktoren und Effektivität präventiver Strategien}

\begin{abstract}
Speziell bei älteren Menschen mit Malignomen ist mit schweren Nebenwirkungen einer Chemotherapie zu rechnen. Wie lässt sich ein Toxizitätsrezidiv vorhersehen und vermeiden, ohne die Dosisintensität aufs Spiel zu setzen?
\end{abstract}

n die prospektive Studie wurden 200 ältere Tumorpatienten ( $\geq 65$ Jahre) vor Beginn ihrer Chemotherapie aufgenommen. Bei Auftreten schwerer Toxizität $(\mathrm{n}=82)$ wurden folgende Parameter erhoben: Dauer und funktionelle Folgen dieser Nebenwirkungen, die durchgeführten Therapiemodifikationen und das Wiederauftreten einer schweren Toxizität. Die durchschnittliche Toxizität des Regimes wurde mithilfe des MAX2Index eingeschätzt, der sich auf die Häufigkeit der Toxizitäten stützt.

Im Mittel erhielten die Patienten (median 73 Jahre) 4,73 Chemotherapiezyklen. Die Daten von 163 Patienten konnten nach einem oder mehr Zyklen ausgewertet werden. Von ihnen erlebten 82 eine schwere Toxizität, bei 10 wurde die Therapie deshalb abgebrochen, bei weiteren 6 erfolgte ein Abbruch aus anderen Gründen, 5 Patienten starben.

61 Patienten bekamen eine weitere Chemotherapie: 20 mit und 41 ohne Dosismodifikation. In der Gruppe ohne Dosismodifikationen erlebten 19 (46\%) einen Toxizitätsrückfall (0 Todesfälle), in der Gruppe mit Modifikationen 7 (35\%; 1 Todesfall).

Dosismodifikationen waren in einer Regressionsanalyse assoziiert mit kurativer Behandlungsintention, stationärer
Aufnahme, länger dauernder Beeinträchtigung der Alltagsaktivitäten (ADL), Verschlechterung der Lebensqualität und Fatigue. Der Faktor ADL zeigte seine Assoziation auch in der multivariaten Analyse ( $p=0,02)$.

Mögliche Risikofaktoren für das Toxizitätsrezidiv waren lediglich der ECOGPerformance-Status und der MAX2Score, letzterer auch in der multivariaten Analyse ( $\mathrm{p}=0,04)$.

Fazit: Wenn schwere Toxizität keine längerdauernde Verschlechterung der ADL bewirkt, entscheiden sich Onkologen häufiger, die Therapie nicht zu modifizieren. Mit geeigneten supportiven Maßnahmen führt dies wohl zu ähnlichen Rückfallrisiken wie bei Patienten mit Modifikation - bei insgesamt niedrigem Mortalitätsrisiko. Brigitte Schalhorn

Extermann M et al. Chemotoxicity recurrence in older patients: Risk factors and effectiveness of preventive strategies - a prospective study. Cancer. 2015;121(17):2984-92. 